\title{
MARGINALIZED PHD FILTERS FOR MULTI-TARGET FILTERING
}

\author{
Yohan Petetin, François Desbouvries \\ Telecom Institute / Telecom SudParis / CITI Department and CNRS UMR 5157 \\ 9 rue Charles Fourier, 91011 Evry, France \\ \{yohan.petetin, francois.desbouvries\}atelecom-sudparis.eu
}

\begin{abstract}
Multi-target filtering aims at tracking an unknown number of targets from a set of observations. The Probability Hypothesis Density (PHD) Filter is a promising solution but cannot be implemented exactly. Suboptimal implementation techniques include Gaussian Mixture (GM) solutions, which hold only in linear and Gaussian models, and Sequential Monte Carlo (SMC) algorithms, which estimate the number of targets and their state parameters for a more general class of models. In this paper, we address the case of Gaussian models where the state can be decomposed into a linear component and a non-linear one, and we show that the use of SMC methods in such models can indeed be reduced. Our technique not only improves the estimate of the number of targets but also that of their state. We finally adapt the technique to linear and Gaussian jump Markov state space systems (JMSS) in order to reduce the intractability of existing solutions, and to JMSS with partially linear and partially non-linear state vector.
\end{abstract}

\section{INTRODUCTION}

The problematic of multi-target filtering consists in estimating random state parameters of an unknown number of targets from a set of observations which are either due to detected targets or are false alarms measurements. The PHD Filter is a recent solution based on the Random Finite Set (RFS) theory [1] and is based on the propagation of the PHD function (or intensity), a positive density function which operates in the single target state space domain and which enables to deduce the number of targets as well as the state parameters of each target.

However, the PHD filter involves the computation of complex integrals and thus the PHD cannot be computed exactly either. In practice, two implementations of the PHD filter, with variants, are popular. On the one hand, GM implementations [2] assume that the PHD is a GM, but require that each target (when it is detected) and associated measurement follow a linear and Gaussian model; on the other hand, SMC implementations of the PHD [3] consist in approximating the PHD by a set of weighted particles and do not require any assumption as regards the dynamics of the targets. Now, multiple object filtering SMC-based solutions inherit the same problems as their

\footnotetext{
We would like to thank the French MOD DGA/MRIS for financial support of the Ph.D. of Y. Petetin.
}

single object filtering counterparts (see e.g. [4]). In particular, the specific difficulties of high dimensional problems get worse in multi target scenarios since one needs to estimate the number of targets in addition to the state parameters.

In particle filtering $(\mathrm{PF})$ this problem can somehow be attenuated when the state can be decomposed into a linear component and a nonlinear one. In this case it is of interest to use marginalized PF (MPF) solutions, which roughly speaking estimate the nonlinear part of the state by PF, and conditionally to that non linear part, the linear one via Kalman filtering (KF) [5] [6]. MPF solutions have recently been adapted to multi-target filtering, with an approach based on data association [7] and another on the Bayesian Multi Target Filter (MTF) [8]. The aim of this paper consists in further adapting the MPF idea, but now to the PHD filter which has the advantage of being more tractable than the Bayesian MTF, and which presents good results in highly cluttered environments [2] [1].

More precisely our contribution is threefold. We first derive an implementation of the PHD filter, the Marginalized PHD Filter (M-PHD), which can be used in a Gaussian but only partially linear model. We next consider linear and Gaussian JMSS in the context of multi-target filtering. In such models a GM implementation of the PHD filter has been proposed [9], but the intractability of the GM solution is overemphasized by the different number of modes taken by the discrete Markov Chain (MC) which models the jumps. So we adapt our M-PHD filter to such models in order to avoid the growth of the mixture due to the number of modes. Finally, we consider Gaussian JMSS but now with partially linear and non-linear substructures, for which we also adapt the M-PHD filter. MPHD filters are validated via simulations. The paper is organized as follows. In section 2 we briefly recall the PHD filter and in section 3 develop M-PHD implementations. Section 4 is devoted to simulations and we end the paper with a conclusion.

\section{THE PHD FILTER}

A PHD is a real and positive function $v(\mathbf{x})$ which has the following property: let $S \subset \mathbb{R}^{m}$, then

$$
\int_{S} v(\mathbf{x}) \mathrm{d} \mathbf{x}=\mathrm{E}(|X \cap S|),
$$


where $|X \cap S|$ is the cardinal of the set of the targets which belong to region $S$. In other words, the integral of PHD $v(\mathbf{x})$ over region $S$ is the expected number of objects in this region.

In this framework, the multi-target filtering problem can be seen as the propagation of the filtering PHD $v_{k \mid k}$ i.e. the conditional PHD at time $k$ given all observations up to time $k$. The so-called PHD filter, which is a set of equations which propagate recursively the PHD, can be derived under the following hypotheses [1]: targets evolve and generate measurements independently of one another; the clutter is independent of measurements due to detected targets; and the clutter and the number of targets follow a Poisson distribution. This last assumption can be relaxed but in this case one needs to propagate the cardinality distribution of the number of targets, which yields the Cardinalized PHD (CPHD) filter [10]. We will just consider the case of the PHD filter in this paper, even though our method can be adapted to the CPHD filter.

Let $f_{k \mid k-1}\left(\mathbf{x}_{k} \mid \mathbf{x}_{k-1}\right)$ be the transition pdf from state $\mathbf{x}_{k-1}$ to $\mathbf{x}_{k} ; g_{k}\left(\mathbf{z}_{k} \mid \mathbf{x}_{k}\right)$ the likelihood of a measurement $\mathbf{z}_{k}$ with state $\mathbf{x}_{k} ; p_{s, k}(\mathbf{x})$ the probability that a target with state parameters $\mathbf{x}$ at time $k-1$ still exists at time $k$; $p_{d, k}(\mathbf{x})$ the probability that a target with state parameters $\mathbf{x}$ is detected at time $k ; \kappa_{k}$ (resp. $\gamma_{k}$ ) the PHD of clutter measurements (birth targets) at time $k$. The propagation of the PHD $v_{k \mid k}(\mathbf{x})$ is the succession of a prediction step and of an updating step (we assume for simplicity, but without loss of generality, that there is not spawning) [1]:

$$
\begin{aligned}
v_{k \mid k-1}(\mathbf{x})= & \int p_{s, k}\left(\mathbf{x}_{k-1}\right) f_{k \mid k-1}\left(\mathbf{x} \mid \mathbf{x}_{k-1}\right) \times \\
& v_{k-1 \mid k-1}\left(\mathbf{x}_{k-1}\right) \mathrm{d} \mathbf{x}_{k-1}+\gamma_{k}(\mathbf{x}), \\
v_{k \mid k}(\mathbf{x})= & {\left[1-p_{d, k}(\mathbf{x})\right] v_{k \mid k-1}(\mathbf{x}) } \\
+ & \sum_{\mathbf{z} \in Z_{k}} \frac{p_{d, k}(\mathbf{x}) g_{k}(\mathbf{z} \mid \mathbf{x}) v_{k \mid k-1}(\mathbf{x})}{\kappa_{k}(\mathbf{z})+\int p_{d, k}(\mathbf{x}) g_{k}(\mathbf{z} \mid \mathbf{x}) v_{k \mid k-1}(\mathbf{x}) \mathrm{d} \mathbf{x}} .
\end{aligned}
$$

\section{M-PHD FILTERS}

\subsection{A brief review of MPF in single object filtering}

Assume that the state vector $\mathbf{x}_{k}$ of a target can be divided into a linear component $\mathbf{x}_{k}^{l}$ (with dimension $m_{l}$ ) and a non linear one $\mathbf{x}_{k}^{n}$ (with dimension $m_{n}$ ) (i.e., $\mathbf{x}_{k}^{l}$ follows a linear dynamics and $\mathbf{x}_{k}^{n}$ a non linear one), and that the relations between these components between time $k$ and $k+1$ and the associated measurement $\mathbf{z}_{k}$ are given by:

$$
\begin{aligned}
\mathbf{x}_{k+1}^{n} & =f_{k}^{n}\left(\mathbf{x}_{k}^{n}\right)+\mathbf{F}_{k}^{n}\left(\mathbf{x}_{k}^{n}\right) \mathbf{x}_{k}^{l}+\mathbf{u}_{k}^{n}, \\
\mathbf{x}_{k+1}^{l} & =f_{k}^{l}\left(\mathbf{x}_{k}^{n}\right)+\mathbf{F}_{k}^{l}\left(\mathbf{x}_{k}^{n}\right) \mathbf{x}_{k}^{l}+\mathbf{u}_{k}^{l}, \\
\mathbf{z}_{k+1} & =h_{k}\left(\mathbf{x}_{k+1}^{n}\right)+\mathbf{H}_{k}\left(\mathbf{x}_{k+1}^{n}\right) \mathbf{x}_{k+1}^{l}+\mathbf{v}_{k},
\end{aligned}
$$

in which $f_{k}^{n}($.$) is a function from \mathbb{R}^{m_{n}}$ to $\mathbb{R}^{m_{n}}, \mathbf{F}_{k}^{n}($.$) is$ a $m_{n} \times m_{l}$ matrix which depends on the components of vector $\mathbf{x}_{k}^{n}$ and which acts on vector $\mathbf{x}_{k}^{l}$ (functions $f_{k}^{l}($.$) ,$ $h_{k}(),. \mathbf{F}_{k}^{l}($.$) and \mathbf{H}_{k}($.$) are defined similarly), and$

$$
\left[\begin{array}{c}
\mathbf{u}_{k}^{n} \\
\mathbf{u}_{k}^{l} \\
\mathbf{v}_{k}
\end{array}\right] \sim \mathcal{N}\left(. ;\left[\begin{array}{l}
\mathbf{0} \\
\mathbf{0} \\
\mathbf{0}
\end{array}\right] ;\left[\begin{array}{ccc}
\mathbf{Q}_{k}^{n} & \left(\mathbf{Q}_{k}^{n l}\right)^{T} & 0 \\
\left(\mathbf{Q}_{k}^{n l}\right) & \mathbf{Q}_{k}^{l} & 0 \\
0 & 0 & \mathbf{R}_{k}
\end{array}\right]\right),
$$

where $\left[\mathbf{u}_{1}^{n}, \mathbf{u}_{1}^{l}\right], \cdots,\left[\mathbf{u}_{k}^{n}, \mathbf{u}_{k}^{l}\right]$ and $\mathbf{v}_{1}, \cdots, \mathbf{v}_{k}$ are independent.

Let us now recall MPF [6] [5] [11] in model (4)-(6). Remember that the goal consists in approximating the posterior density $p\left(\mathbf{x}_{k} \mid \mathbf{z}_{0: k}\right)$. MPF techniques are based on the factorization

$$
p\left(\mathbf{x}_{k}^{l}, \mathbf{x}_{0: k}^{n} \mid \mathbf{z}_{0: k}\right)=\underbrace{p\left(\mathbf{x}_{k}^{l} \mid \mathbf{x}_{0: k}^{n}, \mathbf{z}_{0: k}\right)}_{\mathrm{KF}} \times \underbrace{p\left(\mathbf{x}_{0: k}^{n} \mid \mathbf{z}_{0: k}\right)}_{\mathrm{PF}} .
$$

The second factor $p\left(\mathbf{x}_{0: k}^{n} \mid \mathbf{z}_{0: k}\right)$ is approximated by PF, and since system (4)-(6) becomes a classical state-space model once $\mathbf{x}_{k}^{n}$ is fixed, the first factor $p\left(\mathbf{x}_{k}^{l} \mid \mathbf{x}_{0: k}^{n}, \mathbf{z}_{0: k}\right)$ can be computed exactly via KF. This idea is popular and its interest is enlightened by the Rao-Blackwell theorem applied to sequential filtering.

\subsection{Extension to multi-target filtering : the M-PHD filter}

We now address PHD-based multi-object filtering algorithms. Remember from section 2 that the expected number of targets at time $k$ is given by $\int_{\mathbb{R}^{m}} v_{k \mid k}\left(\mathbf{x}_{k}\right) \mathrm{d} \mathbf{x}_{k}$, where $v_{k \mid k}\left(\mathbf{x}_{k}\right)$ is the a posteriori filtering PHD and that looking for state parameters boils down to finding regions $S \subset \mathbb{R}^{m}$ such that $\int_{S} v_{k \mid k}\left(\mathbf{x}_{k}\right) \mathrm{d} \mathbf{x}_{k}$ is high. Estimating $\int_{S} v_{k \mid k}\left(\mathbf{x}_{k}\right) \mathrm{d} \mathbf{x}_{k}$ over any region $S$ (or over $\mathbb{R}^{m}$ ) is thus essential in the PHD-based approach, since in practice the integral is not directly computable.

We show that in such a model, the integral of the PHD $\int v_{k \mid k}\left(\mathbf{x}_{k}\right) \mathrm{d} \mathbf{x}_{k}$ can be computed as follow:

$$
\int[\underbrace{\left.\int v_{k \mid k}\left(\mathbf{x}_{k}^{n}, \mathbf{x}_{k}^{l}\right) \mathrm{d} \mathbf{x}_{k}^{n}\right]}_{\text {PF implementation }} \mathrm{d} \mathbf{x}_{k}^{l}=\underbrace{\int \hat{v}_{k \mid k}\left(\mathbf{x}_{k}^{l}\right) \mathrm{d} \mathbf{x}_{k}^{l}}_{\text {Computable }} .
$$

We assume now that the probability of survival $p_{s, k}(\mathbf{x})$ $=p_{s, k}\left(\mathbf{x}^{n}\right)$ and the probability of detection $p_{d, k}(\mathbf{x})=$ $p_{d, k}\left(\mathbf{x}^{n}\right)$ depend only on the non linear component. The Gaussian pdf taken in $\mathbf{x}$, with mean $\mathbf{m}$ and covariance $\mathbf{P}$ is noted $\mathcal{N}(\mathbf{x} ; \mathbf{m} ; \mathbf{P})$ and we assume also that $\gamma_{k+1}(\mathbf{x})$ is a mixture

$\gamma_{k+1}(\mathbf{x})=\sum_{i=1}^{N_{\gamma_{k+1}}} w_{\gamma_{k+1}}^{(i)} p_{i}\left(\mathbf{x}^{n}\right) \mathcal{N}\left(\mathbf{x}^{l} ; \mathbf{m}_{\gamma_{k+1}}^{l,(i)}\left(\mathbf{x}^{n}\right) ; \mathbf{P}_{\gamma_{k+1}}^{l,(i)}\right)$ where $p_{i}(\mathbf{x})$ is a pdf for each $i, 1 \leq i \leq N_{\gamma_{k+1}}$.

The key idea of our approach is to assume that at time $k$, the PHD is neither a Gaussian nor a discrete mixture, but a mixture with the following form, hereafter denoted normal discrete $(\mathrm{ND})$ :

$$
\hat{v}_{k \mid k}\left(\mathbf{x}_{k}\right)=\sum_{i=1}^{L_{k}} w_{k \mid k}^{(i)} \mathcal{N}\left(\mathbf{x}_{k}^{l} ; \mathbf{m}_{k \mid k}^{l,(i)} ; \mathbf{P}_{k \mid k}^{l,(i)}\right) \delta_{\mathbf{x}_{k}^{n,(i)}}\left(\mathbf{x}_{k}^{n}\right),
$$

with $\mathbf{x}_{k}=\left[\mathbf{x}_{k}^{l}, \mathbf{x}_{k}^{n}\right]$. As we now see it is possible to propagate an ND approximation of the PHD $v_{k \mid k}\left(\mathbf{x}_{k}\right)$. Our algorithm consists of three steps, described in $\$ 3.2 .1$ 3.2.3 below; the predicting (see \$3.2.1) and updating (see $\S 3.2 .3)$ steps are derived by using conditioning properties in Gaussian pdfs (proofs are omited for lack of space). 


\subsubsection{Prediction}

Let us set $\mathbf{F}_{k}^{l,(i)} \triangleq \mathbf{F}_{k}^{l}\left(\mathbf{x}_{k}^{n,(i)}\right), \mathbf{F}_{k}^{n,(i)} \triangleq \mathbf{F}_{k}^{n}\left(\mathbf{x}_{k}^{n,(i)}\right), f_{k}^{l,(i)}$ $\triangleq f_{k}^{l}\left(\mathbf{x}_{k}^{n,(i)}\right), f_{k}^{n,(i)} \triangleq f_{k}^{n}\left(\mathbf{x}_{k}^{n,(i)}\right)$ and $p_{s, k}^{(i)} \triangleq p_{s, k}\left(\mathbf{x}_{k}^{n,(i)}\right)$. Let $\hat{v}_{k \mid k}$ in (9) be an ND approximation of PHD $v_{k \mid k}$. Then our first step consists in plugging (9) into (2), which yields the following approximation $\tilde{v}_{k+1 \mid k}$ of the predicted PHD $v_{k+1 \mid k}$ :

$$
\begin{aligned}
\tilde{v}_{k+1 \mid k}(\mathbf{x}) & =\sum_{i=1}^{L_{k}} w_{k+1 \mid k}^{(i)} \mathcal{N}\left(\mathbf{x}^{l} ; \mathbf{m}_{k+1 \mid k}^{l,(i)}\left(\mathbf{x}^{n}\right) ; \mathbf{P}_{k+1 \mid k}^{l,(i)}\right) \\
& \times \mathcal{N}\left(\mathbf{x}^{n} ; \mathbf{m}_{k+1 \mid k}^{n,(i)} ; \mathbf{P}_{k+1 \mid k}^{n,(i)}\right)+\gamma_{k+1}(\mathbf{x}),(10)
\end{aligned}
$$

where

$$
\begin{aligned}
w_{k+1 \mid k}^{(i)} & =p_{s, k+1}^{(i)} w_{k \mid k}^{(i)}, \\
\mathbf{m}_{k+1 \mid k}^{n,(i)} & =f_{k}^{n,(i)}+\mathbf{F}_{k}^{n,(i)} \mathbf{m}_{k \mid k}^{l,(i)} \\
\mathbf{P}_{k+1 \mid k}^{n,(i)} & =\mathbf{Q}_{k}^{n}+\mathbf{F}_{k}^{n,(i)} \mathbf{P}_{k \mid k}^{l,(i)} \mathbf{F}_{k}^{n,(i)}, \\
\mathbf{m}_{k+1 \mid k}^{l,(i)}\left(\mathbf{x}_{k+1}^{n}\right) & =\mathbf{A}_{k}^{(i)} \tilde{\mathbf{m}}_{k+1 \mid k}^{l,(i)}\left(\mathbf{x}_{k+1}^{n}\right)+f_{k}^{l,(i)} \\
& +\mathbf{Q}_{k}^{n l}\left(\mathbf{Q}_{k}^{n}\right)^{-1}\left(\mathbf{x}_{k+1}^{n}-f_{k}^{n,(i)}\right), \\
\mathbf{P}_{k+1 \mid k}^{l,(i)} & =\mathbf{Q}_{k}^{l}-\mathbf{Q}_{k}^{n l}\left(\mathbf{Q}_{k}^{n}\right)^{-1} \mathbf{Q}_{k}^{(n l)^{T}} \\
& +\mathbf{A}_{k}^{(i)} \tilde{\mathbf{P}}_{k+1 \mid k}^{l,(i)} \mathbf{A}_{k}^{(i)^{T}}
\end{aligned}
$$

in which $\tilde{\mathbf{m}}_{k+1 \mid k}^{l,(i)}\left(\mathbf{x}^{n}\right), \tilde{\mathbf{P}}_{k+1 \mid k}^{l,(i)}$ and $\mathbf{A}_{k}^{(i)}$ are defined by

$$
\begin{aligned}
\tilde{\mathbf{m}}_{k+1 \mid k}^{l,(i)}\left(\mathbf{x}^{n}\right) & =\mathbf{m}_{k \mid k}^{l,(i)} \\
& +\tilde{\mathbf{K}}_{k+1 \mid k}^{l,(i)}\left[\mathbf{x}^{n}-f_{k}^{n,(i)}-\mathbf{F}_{k}^{n,(i)} \mathbf{m}_{k \mid k}^{l,(i)}\right] \\
\tilde{\mathbf{P}}_{k+1 \mid k}^{l,(i)} & =\left[\mathbf{I}-\tilde{\mathbf{K}}_{k+1 \mid k}^{l,(i)} \mathbf{F}_{k}^{n,(i)}\right] \mathbf{P}_{k \mid k}^{l,(i)}, \\
\mathbf{A}_{k}^{(i)} & =\left[\mathbf{F}_{k}^{l,(i)}-\mathbf{Q}_{k}^{n l}\left(\mathbf{Q}_{k}^{n}\right)^{-1} \mathbf{F}_{k}^{n,(i)}\right]
\end{aligned}
$$

where

$$
\tilde{\mathbf{K}}_{k+1 \mid k}^{l,(i)}=\mathbf{P}_{k \mid k}^{l,(i)} \mathbf{F}_{k}^{n,(i)}\left[\mathbf{F}_{k}^{n,(i)} \mathbf{P}_{k \mid k}^{l,(i)} \mathbf{F}_{k}^{n,(i)}+\mathbf{Q}_{k}^{n}\right]^{-1}
$$

\subsubsection{Discrete Approximation of $\tilde{v}_{k+1 \mid k}\left(\mathbf{x}_{k+1}\right)$}

To propagate the PHD we need to derive a discrete approximation of $\tilde{v}_{k+1 \mid k}\left(\mathbf{x}_{k+1}\right)$. This can be achieved by deriving a discrete approximation of the first mixture in (10) and one of $\gamma_{k}\left(\mathbf{x}_{k+1}\right)$ :

- Sample $\mathbf{x}_{k+1}^{n,(i)} \sim \mathcal{N}\left(\mathbf{x}_{k+1}^{n} ; \mathbf{m}_{k+1 \mid k}^{n,(i)} ; \mathbf{P}_{k+1 \mid k}^{n,(i)}\right)$, for $1 \leq$ $i \leq L_{k}$ (other importance distributions can be chosen, see [3]) and set $\mathbf{m}_{k+1 \mid k}^{l,(i)}=\mathbf{m}_{k+1 \mid k}^{l,(i)}\left(\mathbf{x}_{k+1}^{n,(i)}\right)$;

- Sample $\mathbf{x}_{k+1}^{n,(i)} \sim \sum_{j=1}^{N_{\gamma_{k+1}}} w_{\gamma_{k+1}}^{(j)} p_{j}\left(\mathbf{x}_{k+1}^{n}\right)$ for $L_{k}+$ $1 \leq i \leq L_{k}+L_{\gamma_{k+1}}$, by first sampling index $j^{i} \sim$ $\sum_{j=1}^{N_{\gamma_{k+1}}} \frac{w_{\gamma_{k+1}}^{(j)}}{\sum_{i=1}^{N \gamma_{k+1}} w_{\gamma_{k+1}}^{(i)}} \delta_{j}$ then $\mathbf{x}_{k+1}^{n,(i)} \sim p_{j^{i}}\left(\mathbf{x}_{k+1}^{n}\right)$ and initialize $\mathbf{m}_{k+1 \mid k}^{l,(i)}=\mathbf{m}_{\gamma_{k+1}}^{l,\left(j^{i}\right)}\left(\mathbf{x}_{k+1}^{n,(i)}\right), \mathbf{P}_{k+1 \mid k}^{l,(i)}=$ $\mathbf{P}_{\gamma_{k+1}}^{l,\left(j^{i}\right)}$ and $w_{k+1 \mid k}^{(i)}=\sum_{i=1}^{N_{\gamma_{k+1}}} w_{\gamma_{k+1}}^{(i)} / L_{\gamma_{k+1}}$.

Finally, $\tilde{v}_{k+1 \mid k}$ is approximated by an ND approximation of $L_{k+1 \mid k}=L_{k}+L_{\gamma_{k+1}}$ components.

\subsubsection{Updating}

We set $h_{k}^{(i)} \triangleq h_{k}\left(\mathbf{x}_{k+1}^{n,(i)}\right), \mathbf{H}_{k}^{(i)} \triangleq \mathbf{H}_{k}\left(\mathbf{x}_{k+1}^{n,(i)}\right)$ and $p_{d, k+1}^{(i)} \triangleq$ $p_{d, k+1}\left(\mathbf{x}_{k+1}^{n,(i)}\right)$. It remains to implement equation (3), which finally gives the ND approximation $\hat{v}_{k+1 \mid k+1}$ of $v_{k+1 \mid k+1}$ :

$$
\begin{aligned}
& \hat{v}_{k+1 \mid k+1}(\mathbf{x})=\sum_{i=1}^{L_{k+1 \mid k}}\left[w_{k+1 \mid k+1}^{(i)}(\emptyset) \mathcal{N}\left(\mathbf{x}^{l} ; \mathbf{m}_{k+1 \mid k}^{l,(i)} ; \mathbf{P}_{k+1 \mid k}^{l,(i)}\right)\right. \\
& \left.+\sum_{\mathbf{z} \in Z_{k}} w_{k+1 \mid k+1}^{(i)}(\mathbf{z}) \psi_{k+1 \mid k+1}^{(i)}\left(\mathbf{x}^{l}, \mathbf{z}\right)\right] \delta_{\mathbf{x}_{k+1}^{n,(i)}}\left(\mathbf{x}^{n}\right), \quad \text { (20) }
\end{aligned}
$$

where

$$
\begin{aligned}
\psi_{k+1 \mid k+1}^{(i)}\left(\mathbf{x}^{l}, \mathbf{z}\right) & =\mathcal{N}\left(\mathbf{x}^{l} ; \mathbf{m}_{k+1 \mid k+1}^{l,(i)}(\mathbf{z}) ; \mathbf{P}_{k+1 \mid k+1}^{l,(i)}\right) \\
w_{k+1 \mid k+1}^{(i)}(\emptyset) & =\left(1-p_{d, k+1}^{(i)}\right) w_{k+1 \mid k}^{(i)}, \\
w_{k+1 \mid k+1}^{(i)}(\mathbf{z}) & =\left(p_{d, k+1}^{(i)} q_{k+1}\left(\mathbf{z}, \mathbf{x}_{k+1}^{n,(i)}\right) w_{k+1 \mid k}^{(i)}\right)(\kappa(\mathbf{z}) \\
\left.+\sum_{i=1}^{L_{k+1}} w_{k+1 \mid k}^{(i)} p_{d, k+1}^{(i)} q_{k+1}\left(\mathbf{z}, \mathbf{x}_{k+1}^{n,(i)}\right)\right)^{-1}, & \\
\mathbf{m}_{k+1 \mid k+1}^{l,(i)}(\mathbf{z}) & =\mathbf{m}_{k+1 \mid k}^{l,(i)} \\
+ & \mathbf{K}_{k+1}^{(i)}\left[\mathbf{z}-\mathbf{H}_{k}^{(i)} \mathbf{m}_{k+1 \mid k}^{l,(i)}-h_{k}^{(i)}\right] \\
\mathbf{P}_{k+1 \mid k+1}^{l,(i)} & =\left[\mathbf{I}-\mathbf{K}_{k+1}^{(i)} \mathbf{H}_{k}^{(i)}\right] \mathbf{P}_{k+1 \mid k}^{l,(i)},
\end{aligned}
$$

where

$$
\begin{aligned}
& q_{k+1}\left(\mathbf{z}, \mathbf{x}_{k+1}^{n,(i)}\right)= \\
& \mathcal{N}\left(\mathbf{z} ; h_{k}^{(i)}+\mathbf{H}_{k}^{(i)} \mathbf{m}_{k+1 \mid k}^{l,(i)} ; \mathbf{R}_{k}+\mathbf{H}_{k}^{(i)} \mathbf{P}_{k+1 \mid k}^{l,(i)} \mathbf{H}_{k}^{(i)^{T}}\right), \\
& \mathbf{K}_{k+1}^{(i)}=\mathbf{P}_{k+1 \mid k}^{l,(i)} \mathbf{H}_{k}^{(i)}\left[\mathbf{H}_{k}^{(i)} \mathbf{P}_{k+1 \mid k}^{l,(i)} \mathbf{H}_{k}^{(i)^{T}}+\mathbf{R}_{k}\right]^{-1} .
\end{aligned}
$$

\subsection{Other applications of the M-PHD filter}

We next focus on models where a dependence in a discrete Markov Chain $r_{k}$ is introduced and so we consider the augmented state $\left[\mathbf{x}_{k}, r_{k}\right]$. These models are of practical interest for tracking maneuvering targets.

\subsubsection{Linear and Gaussian JMSS}

The technique described above is adaptable for linear and Gaussian JMSS, described by the following equations:

$$
\begin{aligned}
& \mathbf{x}_{k+1}=\mathbf{F}_{k}\left(r_{k+1}\right) \mathbf{x}_{k}+\mathbf{G}_{k}\left(r_{k+1}\right) \mathbf{u}_{k}, \\
& \mathbf{z}_{k+1}=\mathbf{H}_{k}\left(r_{k+1}\right) \mathbf{x}_{k+1}+\mathbf{B}_{k}\left(r_{k+1}\right) \mathbf{v}_{k}, \\
& r_{k} \text { is a discrete MC, }
\end{aligned}
$$

where $\mathbf{u}_{k} \sim \mathcal{N}\left(. ; \mathbf{0} ; \mathbf{Q}_{k}\right), \mathbf{v}_{k} \sim \mathcal{N}\left(. ; \mathbf{0} ; \mathbf{R}_{k}\right)$ and where $\mathbf{u}_{1}, \cdots, \mathbf{u}_{k}$ and $\mathbf{v}_{1}, \cdots, \mathbf{v}_{k}$ are independent and independent of $r_{1}, \cdots, r_{k}$. We set $\mathbf{Q}_{k}(r)=\mathbf{G}_{k}(r) \mathbf{Q}_{k} \mathbf{G}_{k}(r)^{T}$ and $\mathbf{R}_{k}(r)=\mathbf{B}_{k}(r) \mathbf{R}_{k} \mathbf{B}_{k}(r)^{T}$.

In the GM implementation of the PHD filter [9], even in the particular case where there is no birth nor spawning, if PHD $v_{k \mid k}$ is characterized by $\sum_{r_{k}} J\left(r_{k}\right)$ Gaussians $\left(J\left(r_{k}\right)\right.$ ones for a given mode $\left.r_{k}\right)$ then the predicted PHD 
$v_{k+1 \mid k}\left(\mathbf{x}_{k+1}, r_{k+1}\right)$ requires the computation of $\sum_{r_{k}} J\left(r_{k}\right)$ $\times|r|$ Gaussians $(|r|$ is the number of different modes of MC $\left.r_{n}\right)$, and the updated PHD $v_{k+1 \mid k+1}\left(\mathbf{x}_{k+1}, r_{k+1}\right)$ the computation of $\sum_{r_{k}} J\left(r_{k}\right) \times|r| \times\left[\left|Z_{k+1}\right|+1\right]$ Gaussians.

As we now see, adapting our marginalized solution enables to avoid the growth of the mixture which is due to $|r|$. Let us assume that $\gamma_{k+1}(\mathbf{x}, r)=\pi_{k+1}(r) \tilde{\gamma}_{k+1}(\mathbf{x}, r)$, where

$$
\tilde{\gamma}_{k+1}(\mathbf{x}, r)=\sum_{i=1}^{N_{\gamma_{k+1}}(r)} w_{\gamma_{k+1}}^{(i)}(r) \mathcal{N}\left(\mathbf{x} ; \mathbf{m}_{\gamma_{k+1}}^{(i)}(r) ; \mathbf{P}_{\gamma_{k+1}}^{(i)}(r)\right),
$$

and that the probabilities of survival $p_{s, k+1}(r)$ and of detection $p_{d, k+1}(r)$ only depend on the mode. As in Section 3.2 our algorithm consists of the three following steps.

- First, let

$$
\sum_{i=1}^{L_{k}} w_{k \mid k}^{(i)} \mathcal{N}\left(\mathbf{x}_{k} ; \mathbf{m}_{k \mid k}^{(i)} ; \mathbf{P}_{k \mid k}^{(i)}\right) \delta_{r_{k}^{(i)}}\left(r_{k}\right)
$$

be an ND approximation of $v_{k \mid k}$. Then we compute the approximation $\tilde{v}_{k+1 \mid k}(\mathbf{x}, r)$ of $v_{k+1 \mid k}$, where

$$
\begin{aligned}
& \tilde{v}_{k+1 \mid k}(\mathbf{x}, r)=\sum_{i=1}^{L_{k}} w_{k \mid k}^{(i)} p_{s, k+1}\left(r_{k}^{(i)}\right) p\left(r \mid r_{k}^{(i)}\right) \times \\
& \mathcal{N}\left(\mathbf{x}_{k+1} ; \mathbf{m}_{k+1 \mid k}^{(i)}(r) ; \mathbf{P}_{k+1 \mid k}^{(i)}(r)\right)+\gamma_{k+1}(\mathbf{x}, r),
\end{aligned}
$$

where

$$
\begin{aligned}
\mathbf{m}_{k+1 \mid k}^{(i)}(r) & =\mathbf{F}_{k}(r) \mathbf{m}_{k \mid k}^{(i)}, \\
\mathbf{P}_{k+1 \mid k}^{(i)}(r) & =\mathbf{F}_{k}(r) \mathbf{P}_{k \mid k}^{(i)} \mathbf{F}_{k}(r)^{T}+\mathbf{Q}_{k}(r) .
\end{aligned}
$$

- Next one can derive an ND approximation of $v_{k+1 \mid k}$ by using a procedure similar to that described in §3.2.2: sample $r_{k+1}^{(i)} \sim p\left(r_{k+1} \mid r_{k}^{(i)}\right)$ for $1 \leq i \leq L_{k}$ and derive an ND approximation of $L_{\gamma_{k+1}}$ components of $\gamma_{k+1}\left(\mathbf{x}_{k+1}, r_{k+1}\right)$. This provides an ND approximation of $L_{k}+L_{\gamma_{k+1}}$ components of PHD $v_{k+1 \mid k}$.

- Finally, the updating step (which enables to compute $\hat{v}_{k+1 \mid k+1}$ ) is identical to that of section 3.2.3, except that $\mathbf{x}_{k+1}^{n,(i)}$ should be replaced by $r_{k+1}^{(i)}, \mathbf{R}_{k}$ by $\mathbf{R}_{k}\left(r_{k+1}^{(i)}\right)$ and $h_{k}^{(i)}=0$.

\subsubsection{Gaussian JMSS with partially linear and partially non-linear sub-structures}

Finally we adapt the M-PHD algorithm proposed in Section 3.2 to a more general class of models (we note $\xi_{k}=$ $\left[\mathbf{x}_{k}^{n}, r_{k+1}\right]$ and $\left.\psi_{k}=\left[\mathbf{x}_{k+1}^{n}, r_{k+1}\right]\right)$ :

$$
\begin{aligned}
\mathbf{x}_{k+1}^{n} & =f_{k}^{n}\left(\xi_{k}\right)+\mathbf{F}_{k}^{n}\left(\xi_{k}\right) \mathbf{x}_{k}^{l}+\mathbf{G}_{k}^{n}\left(\xi_{k}\right) \mathbf{u}_{k}^{n}, \\
\mathbf{x}_{k+1}^{l} & =f_{k}^{l}\left(\xi_{k}\right)+\mathbf{F}_{k}^{l}\left(\xi_{k}\right) \mathbf{x}_{k}^{l}+\mathbf{G}_{k}^{l}\left(\xi_{k}\right) \mathbf{u}_{k}^{l}, \\
\mathbf{z}_{k+1} & =h_{k}\left(\psi_{k}\right)+\mathbf{H}_{k}\left(\psi_{k}\right) \mathbf{x}_{k+1}^{l}+\mathbf{B}_{k}\left(\psi_{k}\right) \mathbf{v}_{k}, \\
r_{k} & \text { is a discrete } \mathbf{M C},
\end{aligned}
$$

where $\left[\mathbf{u}_{1}^{n}, \mathbf{u}_{1}^{l}\right] \cdots,\left[\mathbf{u}_{k}^{n}, \mathbf{u}_{k}^{l}\right]$ and $\mathbf{v}_{1}, \cdots, \mathbf{v}_{k}$ are independent and independent of $r_{1}, \cdots, r_{k}$, and are defined as in (7). We note $\mathbf{Q}_{k}^{n}\left(\mathbf{x}^{n}, r\right)=\mathbf{G}_{k}^{n}\left(\mathbf{x}^{n}, r\right) \mathbf{Q}_{k}^{n} \mathbf{G}_{k}^{n}\left(\mathbf{x}^{n}, r\right)^{T}$, $\mathbf{Q}_{k}^{l}\left(\mathbf{x}^{n}, r\right)=\mathbf{G}_{k}^{l}\left(\mathbf{x}^{n}, r\right) \mathbf{Q}_{k}^{l} \mathbf{G}_{k}^{l}\left(\mathbf{x}^{n}, r\right)^{T}, \mathbf{Q}_{k}^{n l}\left(\mathbf{x}^{n}, r\right)=$ $\mathbf{G}_{k}^{l}\left(\mathbf{x}^{n}, r\right) \mathbf{Q}_{k}^{n l} \mathbf{G}_{k}^{n}\left(\mathbf{x}^{n}, r\right)^{T}$ and $\mathbf{R}_{k}\left(\mathbf{x}^{n}, r\right)=\mathbf{B}_{k}\left(\mathbf{x}^{n}, r\right)$ $\mathbf{R}_{k} \mathbf{B}_{k}\left(\mathbf{x}^{n}, r\right)^{T}$. This model is a generalization of that described by (4)-(6) so we adapt the approach of Section 3.2. We assume that $\gamma_{k+1}(\mathbf{x}, r)=\pi_{k+1}(r) \times \tilde{\gamma}_{k+1}(\mathbf{x}, r)$ where, for given pdfs $p_{i}\left(\mathbf{x}^{n} \mid r\right)$ depending on $r$,

$$
\begin{aligned}
& \tilde{\gamma}_{k+1}(\mathbf{x}, r)=\sum_{i=1}^{N_{\gamma_{k+1}}(r)} w_{\gamma_{k+1}}^{(i)}(r) p_{i}\left(\mathbf{x}^{n} \mid r\right) \times \\
& \mathcal{N}\left(\mathbf{x}^{l} ; \mathbf{m}_{\gamma_{k+1}}^{(i)}\left(\mathbf{x}^{n}, r\right) ; \mathbf{P}_{\gamma_{k+1}}^{(i)}(r)\right),
\end{aligned}
$$

and that the probabilities of survival $p_{s, k+1}\left(\mathbf{x}^{n}, r\right)$ and of detection $p_{d, k+1}\left(\mathbf{x}^{n}, r\right)$ depend only on the mode and on the non-linear component. Let us assume also that at time $k$ an ND approximation of the PHD at time $k$ is given by

$$
\begin{aligned}
& \hat{v}_{k \mid k}\left(\mathbf{x}_{k}, r_{k}\right)= \\
& \sum_{i=1}^{L_{k}} w_{k \mid k}^{(i)} \mathcal{N}\left(\mathbf{x}_{k}^{l} ; \mathbf{m}_{k \mid k}^{l,(i)} ; \mathbf{P}_{k \mid k}^{l,(i)}\right) \delta_{\mathbf{x}_{k}^{n,(i)}, r_{k}^{(i)}}\left(\mathbf{x}_{k}^{n}, r_{k}\right) .
\end{aligned}
$$

Then our algorithm consists of the three following steps.

- The approximated predicted PHD is given by

$$
\begin{aligned}
\tilde{v}_{k+1 \mid k}(\mathbf{x}, r) & =\sum_{i=1}^{L_{k}} w_{k+1 \mid k}^{(i)} p\left(r \mid r_{k}^{(i)}\right) \Phi_{i}\left(\mathbf{x}^{l}, \mathbf{x}^{n}, r_{k+1}\right) \\
& +\gamma_{k+1}(\mathbf{x}, r),
\end{aligned}
$$

where

$$
\begin{aligned}
\Phi_{i}\left(\mathbf{x}^{l}, \mathbf{x}^{n}, r\right) & =\mathcal{N}\left(\mathbf{x}^{l} ; \mathbf{m}_{k+1 \mid k}^{l,(i)}\left(\mathbf{x}^{n}, r\right) ; \mathbf{P}_{k+1 \mid k}^{l,(i)}(r)\right) \\
& \times \mathcal{N}\left(\mathbf{x}^{n} ; \mathbf{m}_{k+1 \mid k}^{n,(i)}(r) ; \mathbf{P}_{k+1 \mid k}^{n,(i)}(r)\right)
\end{aligned}
$$

and where $\mathbf{m}_{k+1 \mid k}^{l,(i)}\left(\mathbf{x}^{n}, r\right), \mathbf{P}_{k+1 \mid k}^{l,(i)}(r), \mathbf{m}_{k+1 \mid k}^{n,(i)}(r)$, $\mathbf{P}_{k+1 \mid k}^{n,(i)}(r)$ and $w_{k+1 \mid k}^{(i)}$ are given by (11)-(19), in which we introduce the dependency in $r$. More precisely, $f_{k}^{n,(i)}, f_{k}^{l,(i)}, \mathbf{F}_{k}^{n,(i)}$ and $\mathbf{F}_{k}^{l,(i)}$ have to be replaced respectively by $f_{k}^{n}\left(\mathbf{x}_{k}^{n,(i)}, r\right), f_{k}^{l}\left(\mathbf{x}_{k}^{n,(i)}, r\right)$, $\mathbf{F}_{k}^{n}\left(\mathbf{x}_{k}^{n,(i)}, r\right)$ and $\mathbf{F}_{k}^{l}\left(\mathbf{x}_{k}^{n,(i)}, r\right)$; and $\mathbf{Q}_{k}^{n}, \mathbf{Q}_{k}^{n l}$ and $\mathbf{Q}_{k}^{l}$ respectively by $\mathbf{Q}_{k}^{n}\left(\mathbf{x}_{k}^{n,(i)}, r\right), \mathbf{Q}_{k}^{n l}\left(\mathbf{x}_{k}^{n,(i)}, r\right)$ and $\mathbf{Q}_{k}^{l}\left(\mathbf{x}_{k}^{n,(i)}, r\right)$;

- Next a discrete approximation of $\tilde{v}_{k+1 \mid k}\left(\mathbf{x}_{k+1}, r_{k+1}\right)$ is obtained by sampling particles $\left[\mathbf{x}_{k+1}^{n,(i)}, r_{k+1}^{(i)}\right]$ in augmented dimension, according to pdf $p\left(r_{k+1} \mid r_{k}^{(i)}\right)$ $\times \mathcal{N}\left(\mathbf{x} ; \mathbf{m}_{k+1 \mid k}^{n,(i)}(r) ; \mathbf{P}_{k+1 \mid k}^{n,(i)}(r)\right)$ for $1 \leq i \leq L_{k}$, and by deriving a discrete approximation of $\gamma_{k+1}(\mathbf{x}$, $r)$;

- Finally, the updating step is also given by (20)-(27), except that $h_{k}^{(i)}, \mathbf{H}_{k}^{(i)}, \mathbf{R}_{k}$ and $p_{d, k+1}^{(i)}$ are replaced by $h_{k}\left(\mathbf{x}_{k+1}^{n,(i)}, r_{k+1}^{(i)}\right), \mathbf{H}_{k}\left(\mathbf{x}_{k+1}^{n,(i)}, r_{k+1}^{(i)}\right), \mathbf{R}_{k}\left(\mathbf{x}_{k+1}^{n,(i)}\right.$, $\left.r_{k+1}^{(i)}\right)$ and $p_{d, k+1}\left(\mathbf{x}_{k+1}^{n,(i)}, r_{k+1}^{(i)}\right)$. 


\section{SIMULATIONS}

In this section we compare the M-PHD and SMC implementations of the PHD filter. Because both methods rely on SMC methods, the same mechanisms are used to initialize particles, extract the state parameters of the target and resample particles (these mechanisms are described in [12]).

\subsection{Optimal Subpattern Assignment (OSPA) metric}

To compare both algorithms we use the OSPA metric, a metric adapted to the multi-target filtering problem [13]. Let $X=\left\{x_{1}, \ldots, x_{m}\right\}$ and $Y=\left\{y_{1}, \ldots, y_{n}\right\}$ be two finite sets; here $X$ (resp. $Y$ ) represents the estimated (resp. true) finite set of targets. For $c>0$ let $d^{(c)}(x, y)=$ $\min (c,\|x-y\|)(\|$.$\| denotes Euclidean norm) and let$ $\Pi_{n}$ be the set of permutations on $\{1,2, \ldots, n\}$. Then for $1 \leq p<+\infty$, the OSPA metric is defined by:

$$
\begin{aligned}
& \bar{d}_{p}^{c}(X, Y) \triangleq \\
& \left(\frac{1}{n}\left(\min _{\pi \in \Pi_{n}} \sum_{i=1}^{m} d^{(c)}\left(x_{i}, y_{\pi(i)}\right)^{p}+c^{p}(n-m)\right)\right)^{\frac{1}{p}}
\end{aligned}
$$

if $m \leq n$, and $\bar{d}_{p}^{c}(X, Y) \triangleq \bar{d}_{p}^{c}(Y, X)$ if $m>n$. In our simulations, we take $c=100$ and $p=2$.

\subsection{Simulations in the Range Bearing Tracking Prob- lem}

We address the range-bearing tracking problem which consists in tracking the Cartesian coordinates of targets from angle and range measurements. It is illustrated by the following equations:

$$
\begin{aligned}
& {\left[\begin{array}{c}
p_{x} \\
\dot{p}_{x} \\
p_{y} \\
\dot{p}_{y}
\end{array}\right]_{k+1}=\left[\begin{array}{cccc}
1 & T & 0 & 0 \\
0 & 1 & 0 & 0 \\
0 & 0 & 1 & T \\
0 & 0 & 0 & 1
\end{array}\right]\left[\begin{array}{c}
p_{x} \\
\dot{p}_{x} \\
p_{y} \\
\dot{p}_{y}
\end{array}\right]_{k}+\mathbf{u}_{k}} \\
& {\left[\begin{array}{c}
z_{b} \\
z_{r}
\end{array}\right]_{k+1}=\left[\begin{array}{c}
\tan ^{-1}\left(p_{y} / p_{x}\right) \\
\sqrt{p_{x}^{2}+p_{y}^{2}}
\end{array}\right]_{k+1}+\mathbf{v}_{k}}
\end{aligned}
$$

with $\mathbf{u}_{k} \sim \mathcal{N}(. ; 0 ; \mathbf{Q})$ and $\mathbf{v}_{k} \sim \mathcal{N}(. ; 0 ; \mathbf{R})$.

This model is a particular case of the more general model presented in Section 3.1, since $f_{k}^{l}\left(\mathbf{x}_{k}^{n}\right)=0, \mathbf{H}_{k}^{l}\left(\mathbf{x}_{k}^{n}\right)$ $=0$ and matrices $\mathbf{F}_{k}^{n}$ and $\mathbf{F}_{k}^{l}$ do not depend on $\mathbf{x}_{k}^{n}$. Note that measurements do not bring any information on the linear component $\left(\mathbf{H}_{k}^{l}\left(\mathbf{x}_{k}^{n}\right)=0\right)$. So if the SMC implementation is used some particles can have well estimated positions, but estimated velocities which do not match the true velocity of the targets. If velocities are not well estimated at time $k$, it can be difficult to properly predict the targets positions at time $k+1$.

We next test the behavior of the algorithms when the velocity of a target can evolve with time. So we generate trajectories from model (45) with

$$
\mathbf{Q}=\sigma_{v}^{2}\left(\begin{array}{cccc}
\frac{T^{3}}{3} & 0 & 0 & 0 \\
0 & T & 0 & 0 \\
0 & 0 & \frac{T^{3}}{3} & 0 \\
0 & 0 & 0 & T
\end{array}\right) \text { and } \mathbf{R}=\left(\begin{array}{cc}
\sigma_{b}^{2} & 0 \\
0 & \sigma_{r}^{2}
\end{array}\right)
$$

We take $\sigma_{b}=\pi / 180 \mathrm{rad}$ and $\sigma_{r}=1 \mathrm{~m}$, so the likelihood is not too tight. A mean of $M=80$ clutter measurements are generated uniformly on $[0, \pi / 2] \times[-1500,2500]$. Figure 1 displays a realization of such a scenario, and indeed for a given coordinate, velocities of a target can be positive or negative, which means that the targets manoeuvre and change of direction.

We run both algorithms 500 times in this scenario. We use $N_{p}=200$ particles for each persistent target and $N_{b}=10$ particles for potential birth targets. Fig. 2 displays the (averaged) OSPA distance and the estimator of the number of targets. As we see, the gap between the MPHD and SMC-PHD algorithm is very large, and this is due to the fact that the number of targets is badly estimated when the SMC-PHD algorithm is used. The reason why is that such a model is indeed challenging for the SMCPHD filter. Since $\mathbf{Q}^{n l}=0$, each component of particles $\mathbf{x}_{k+1}^{(i)}$ is sampled independently given the previous particle $\mathbf{x}_{k}^{(i)}$. Consequently, the linear and non-linear components of particle $\mathbf{x}_{k+1}^{(i)}$ can be inconsistent given the previous one $\mathbf{x}_{k}^{(i)}$. This cannot happen with the M-PHD: even if $\mathbf{Q}^{n l}=$ 0 , the velocity components of the M-PHD are predicted according to the sampled particles. Indeed, according to (14), we have $\mathbf{m}_{k+1 \mid k}^{l,(i)}\left(\mathbf{x}_{k+1}^{n,(i)}\right)=\mathbf{F}_{k}^{l} \tilde{\mathbf{m}}_{k+1 \mid k}^{l,(i)}\left(\mathbf{x}_{k+1}^{n,(i)}\right)$, where $\tilde{\mathbf{m}}_{k+1 \mid k}^{l,(i)}($.$) is given by (16) and depends on \mathbf{x}_{k+1}^{n,(i)}, \mathbf{m}_{k \mid k}^{l,(i)}$ and $\mathbf{x}_{k}^{n,(i)}$. It was also experimented that both algorithms give the same results when the number of particles for the classical SMC implementation is increased to $N_{b}=40$ and $N_{p}=500$. Finally, we perform an experiment where the measurements due to detected targets are very informative $\left(\sigma_{b}=0.001 \mathrm{rad}\right.$ and $\left.\sigma_{r}=1 \mathrm{~m}\right)$, and the mean of clutter measurements is $M=50$. This experiment is challenging for the SMC implementation. We average the OSPA distance over time and we find approximately 15 for the M-PHD and 25 for the SMC-PHD.

\section{CONCLUSION}

In this paper we proposed a Marginalized implementation of the PHD filter for models which present state dynamics which are partially linear. This implementation enables to reduce the dimension of MC samples in the estimation of the PHD, which improves the estimation of both the number of targets and of the state parameters of each target. Our algorithm was extended to Gaussian JMSS with linear or partially linear state dynamics. We finally performed some simulations and compared our M-PHD algorithm against the classical SMC based PHD filter. The M-PHD algorithm outperforms the SMC based PHD filter when the model is informative or if knowledge about the dynamic model of targets is unspecified. 


\section{REFERENCES}

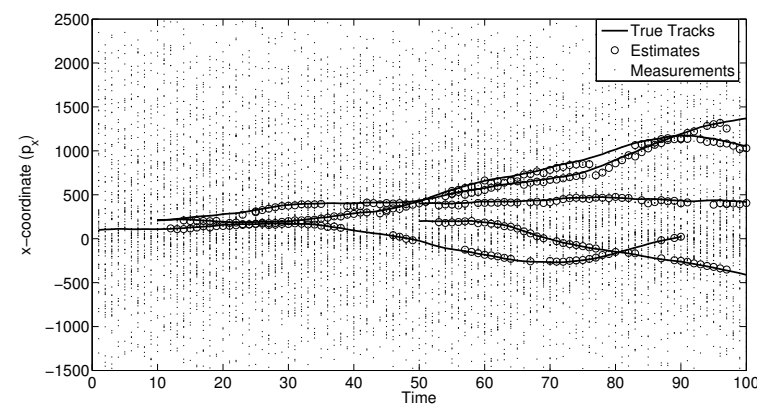

(a)

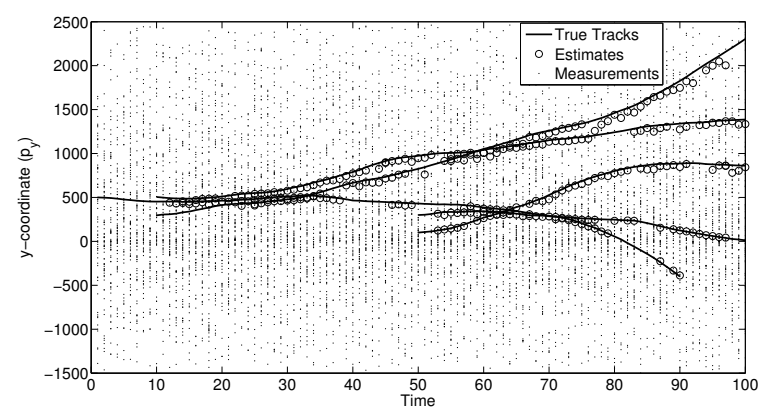

(b)

Fig. 1. Measurements, true target positions and M-PHD filter estimates

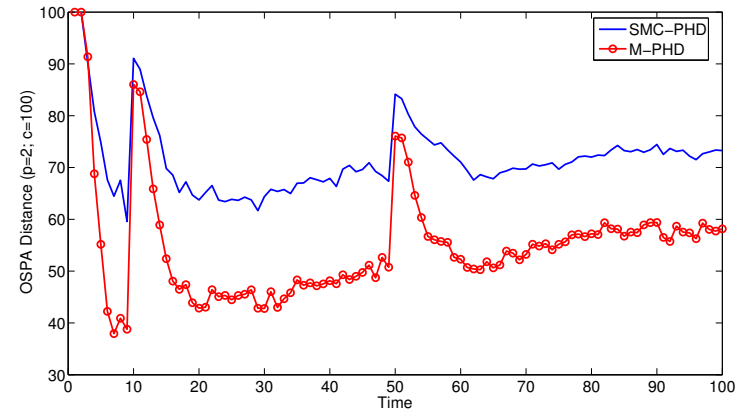

(a)

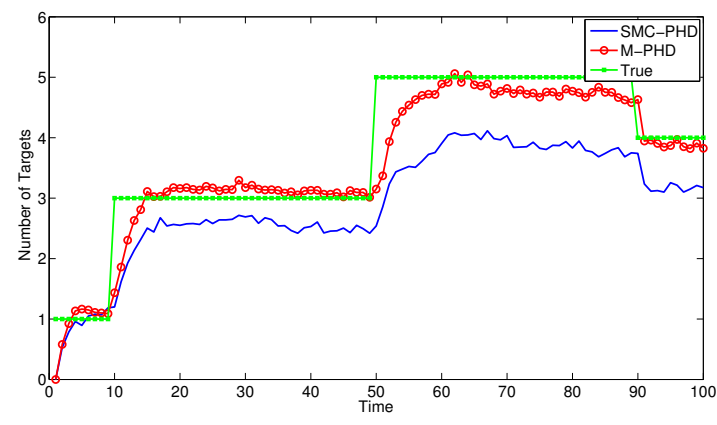

(b)

Fig. 2. OSPA Distance (a) and mean of the estimator of the number of targets (b)
[1] R. Mahler, "Multitarget Bayes Filtering via FirstOrder Multitarget Moments," IEEE Transactions on Aerospace and Electronic Systems, vol. 39, no. 4, October 2003.

[2] B. Vo and W. Ma, "The Gaussian Mixture Probability Hypothesis Density Filter," IEEE transactions on Signal Processing, vol. 54, pp. 4091-4104, November 2006.

[3] B. Vo, S. Singh, and A. Doucet, "Sequential Monte Carlo methods for multi-target filtering with Random Finite Sets," IEEE Transactions on Aerospace and Electronic Systems, vol. 41, 2005.

[4] A. Doucet, N. de Freitas, and N. Gordon, Eds., Sequential Monte Carlo Methods in Practice, ser. Statistics for Engineering and Information Science. New York: Springer Verlag, 2001.

[5] R. Chen and J. S. Liu, "Mixture Kalman Filters," J. R. Statist. Soc. B, vol. 62, pp. 493-508, 2000.

[6] T. Schön, F. Gustafsson, and P.-J. Nordlund, "Marginalized particle filters for mixed linear nonlinear state-space models," IEEE Trans. on Signal Processing, vol. 53, pp. 2279-2289, 2005.

[7] S. Särkkä, A. Vehtari, and J. Lampinen, "Raoblackwellized particle filter for multiple target tracking," Information Fusion Journal, vol. 8, 2007.

[8] M. Vihola, "Rao-blackwellised particle filtering in random set multitarget tracking," IEEE Transactions on Aerospace and Electronic Systems, 2006.

[9] A. Pasha, B.-N. Vo, H. Tuan, and W.-K. Ma, "A Gaussian Mixture PHD Filter for Jump Markov System Models," IEEE Transactions on Aerospace and Electronic Systems, vol. 45, no. 3, pp. 919-936, 2009.

[10] R. Mahler, "A theory of PHD filters of higher order in target number," IEEE Transactions on Aerospace and Electronic Systems , pp. 1523 -1543, October 2007.

[11] A. Doucet, N. J. Gordon, and V. Krishnamurthy, "Particle filters for state estimation of jump Markov linear systems," IEEE Transactions on Signal Processing, vol. 49, no. 3, pp. 613-24, March 2001.

[12] B. Ristic, D. Clark, and B. Vo, "Improved SMC implementation of the PHD Filter," in Proceedings of the 13th International Conference on Information Fusion, 2010.

[13] D. Schuhmacher, B.-T. Vo, and B.-N. Vo, "A consistent metric for performance evaluation of multiobject filters," IEEE Transactions on Signal Processing, vol. 56, no. 8, pp. 3447-3457, August 2008. 